\title{
Evidence for Defects in the Trafficking and Translocation of GLUT4 Glucose Transporters in Skeletal Muscle as a Cause of Human Insulin Resistance
}

\author{
W. Timothy Garvey, ${ }^{\star}$ Lidia Maianu, ${ }^{\star}$ Ju-Hong Zhu, ${ }^{\star}$ Ginger Brechtel-Hook, ${ }^{\ddagger}$ Penny Wallace, ${ }^{\star}$ and Alain D. Baron ${ }^{\ddagger}$ \\ $*$ Department of Medicine, Medical University of South Carolina and Ralph H. Johnson Veterans Affairs Medical Center, Charleston, \\ South Carolina 29425; and ${ }^{\ddagger}$ Department of Medicine, Indiana University School of Medicine and Richard L. Roudebush Veterans Affairs \\ Medical Center, Indianapolis, Indiana 46202
}

\section{Abstract}

Insulin resistance is instrumental in the pathogenesis of type 2 diabetes mellitus and the Insulin Resistance Syndrome. While insulin resistance involves decreased glucose transport activity in skeletal muscle, its molecular basis is unknown. Since muscle GLUT4 glucose transporter levels are normal in type 2 diabetes, we have tested the hypothesis that insulin resistance is due to impaired translocation of intracellular GLUT4 to sarcolemma. Both insulin-sensitive and insulin-resistant nondiabetic subgroups were studied, in addition to type 2 diabetic patients. Biopsies were obtained from basal and insulin-stimulated muscle, and membranes were subfractionated on discontinuous sucrose density gradients to equilibrium or under nonequilibrium conditions after a shortened centrifugation time. In equilibrium fractions from basal muscle, GLUT4 was decreased by 25-29\% in both 25 and $28 \%$ sucrose density fractions and increased twofold in both the $32 \%$ sucrose fraction and bottom pellet in diabetics compared with insulin-sensitive controls, without any differences in membrane markers (phospholemman, phosphalamban, dihydropyridine-binding complex $\alpha-1$ subunit). Thus, insulin resistance was associated with redistribution of GLUT4 to denser membrane vesicles. No effects of insulin stimulation on GLUT4 localization were observed. In non-equilibrium fractions, insulin led to small GLUT4 decrements in the 25 and $28 \%$ sucrose fractions and increased GLUT4 in the $32 \%$ sucrose fraction by 2.8 -fold over basal in insulin-sensitive but only by 1.5 -fold in both insulinresistant and diabetic subgroups. The GLUT4 increments in the $32 \%$ sucrose fraction were correlated with maximal in vivo glucose disposal rates $(r=+0.51, P=0.026)$, and, therefore, represented GLUT4 recruitment to sarcolemma or a quantitative marker for this process. Similar to GLUT4, the insulin-regulated aminopeptidase (vp165) was redistributed to a dense membrane compartment and did not translocate in response to insulin in insulin-resistant subgroups.

In conclusion, insulin alters the subcellular localization of GLUT4 vesicles in human muscle, and this effect is im-

Address correspondence to Dr. W. Timothy Garvey, Division of Endocrinology, Diabetes, and Medical Genetics, Medical University of South Carolina, 171 Ashley Avenue, Charleston, SC 29425. Phone: 843-953-6478; FAX: 843-953-6480; E-mail: garveywt@musc.edu

Received for publication 20 August 1997 and accepted in revised form 24 March 1998.

The Journal of Clinical Investigation

Volume 101, Number 11, June 1998, 2377-2386

http://www.jci.org paired equally in insulin-resistant subjects with and without diabetes. This translocation defect is associated with abnormal accumulation of GLUT4 in a dense membrane compartment demonstrable in basal muscle. We have previously observed a similar pattern of defects causing insulin resistance in human adipocytes. Based on these data, we propose that human insulin resistance involves a defect in GLUT4 traffic and targeting leading to accumulation in a dense membrane compartment from which insulin is unable to recruit GLUT4 to the cell surface. (J. Clin. Invest. 1998. 101:2377-2386.) Key words: insulin resistance • type 2 diabetes mellitus • insulin-regulated aminopeptidase (vp165) • vesicle trafficking • GLUT4

\section{Introduction}

Insulin resistance is key to the pathogenesis of type 2 diabetes mellitus and is also a risk factor associated with hypertension and cardiovascular disease $(1,2)$. The clinical manifestations of insulin resistance, glucose intolerance and hyperinsulinemia, are the consequence of the inability of insulin to stimulate glucose uptake in such insulin target tissues as muscle and fat. The underlying defect must be expressed in skeletal muscle, which accounts for $70-80 \%$ of insulin-stimulated glucose uptake in vivo (3), and current data point to reduced glucose transport activity, the rate-limiting step for glucose uptake (4). Hyperinsulinemic glucose clamp studies and limb balance studies have consistently demonstrated impaired stimulation of muscle glucose uptake in type 2 diabetes (1); however, these techniques do not discriminate whether this is because of a reduction in transport activity or intracellular glucose metabolism. In fact, Bonnadonna et al., using a novel isotope tracer and limb balance approach (5), and Kelley et al. (6), analyzing rate constants for $\left[{ }^{18} \mathrm{~F}\right] 2$-deoxyglucose uptake during positron emission scanning, have provided evidence for separate defects in both transport and glucose phosphorylation in type 2 diabetes. However, under conditions of stimulated glucose flux, investigators have found that free glucose and glucose-6-phosphate do not accumulate in skeletal muscle $(4,7-9)$ and that metabolism within intracellular pathways can be normalized (10-12). These latter data indicate that transport system defects likely predominate in mediating insulin resistance. Most compelling are ex vivo studies that show that the ability of insulin to stimulate glucose transport is markedly impaired in muscle strips and adipocytes removed from insulin resistant patients (13-16).

The molecular basis of insulin resistance remains unclear. Insulin stimulates glucose transport via translocation of GLUT4 glucose transporter proteins from an intracellular depot to the cell surface. In fat cells, GLUT4 expression is markedly reduced in type 2 diabetes and obesity (15). While other defects may exist in insulin action pathways, GLUT4 depletion appears to be the major cause of insulin resistance in fat because of the profound degree to GLUT4 loss and its central role in 
transport stimulation. However, in skeletal muscle, GLUT4 levels were found to be normal in multiple insulin-resistant disease states, including obesity, type 2 diabetes, impaired glucose tolerance, and gestational diabetes $(17,18)$. This has given rise to the inference that defects in GLUT4 translocation cause insulin resistance in muscle $(17,18)$, and supportive data are available $(6,19)$. Such an abnormality could arise from defects in insulin signal transduction or defects in GLUT4 traffic and targeting that lie intrinsic to the glucose transport effector system. Investigators have demonstrated that insulin receptor tyrosine kinase activity, together with downstream signaling events (for example, insulin receptor substrate 1 phosphorylation, phosphatidylinositol-3 kinase activation), are reduced in skeletal muscle (20). Thus, one potential cause of human insulin resistance is a defect in insulin signaling that impairs GLUT4 translocation in muscle.

The presence of signaling defects does not exclude the possibility that critical defects could lie within the glucose transport apparatus. It has become clear that intracellular sequestration and translocation of GLUT4 are regulated by motifs intrinsic to the GLUT4 molecule (21) together with a host of proteins directing vesicle traffic (22-25). The complexity of this system provides multiple potential loci for defects that could interrupt insulin-mediated GLUT4 translocation, and this has increased scientific interest in this area. In adipocytes from women with gestational diabetes, we have shown that impaired GLUT4 translocation was associated with redistribution of GLUT4 to dense membrane vesicles in basal cells suggestive of a defect in transporter trafficking or targeting (16). However, to date, there is no evidence in muscle that defects in GLUT4 trafficking operate as a cause of insulin resistance. In this study, we used a biochemical approach involving subcellular membrane subfractionation of muscle, and, for the first time, implicate defects in GLUT4 traffic and targeting as a cause of impaired GLUT4 translocation and insulin resistance in subjects with and without type 2 diabetes.

\section{Methods}

\section{Subjects}

Table I shows clinical characteristics of the study groups. We studied subjects with normal oral glucose tolerance and patients with type 2 diabetes mellitus (26). Muscle biopsies were processed using two protocols (protocols 1 and 2) based on differences in membrane subfrac- tionation (see below). The nondiabetic subjects in protocol 2 were categorized as insulin sensitive or insulin resistant based on maximally insulin-stimulated glucose uptake rates $>$ or $<15 \mathrm{mg} / \mathrm{kg} / \mathrm{min}$, respectively, during hyperinsulinemic euglycemic clamp studies. All type 2 diabetic patients were being treated with sulfonylurea oral hypoglycemic agents, and were withdrawn from therapy for at least $3 \mathrm{wk}$ and followed on an outpatient basis. All subjects were chemically euthyroid and without renal, hepatic, or cardiac disease. No subject was ingesting pharmacological agents known to affect carbohydrate homeostasis.

\section{In vivo studies}

After we obtained informed consent, we studied nondiabetic and untreated diabetic subjects on a metabolic ward where they remained active. The subjects were allowed to equilibrate on a weight-maintenance diet $(28-32 \mathrm{kcal} / \mathrm{kg} / \mathrm{d})$, consisting of $50 \%$ carbohydrate, $35 \%$ fat, and $15 \%$ protein. We performed standard 75 -g oral glucose tolerance tests (26). We assessed peripheral insulin sensitivity (Table I) using the hyperinsulinemic euglycemic glucose clamp technique as previously described $(27,28)$. During continuous insulin infusion $(400$ $\mathrm{mU} / \mathrm{m}^{2} / \mathrm{min}$ ), the overall glucose disposal rate was calculated on the basis of the glucose infusion rate corrected for changes in glucose pool size, assuming a distribution volume of $19 \%$ body weight and pool fraction of 0.65 . Hepatic glucose output has been shown to be completely suppressed at the high maximally effective insulin concentrations achieved during the clamp (27). During the steady-state period, the serum glucose was maintained at $90 \mathrm{mg} / \mathrm{dl}$ with a coefficient of variation $<4 \%$ by monitoring serum glucose levels at 5 -min intervals and adjusting the infusion rate of a $20 \%$ dextrose solution. Glucose uptake rates were normalized for lean body mass (excluding bone mass) determine by whole-body, dual-energy x-ray absorptiometry scanning.

We performed percutaneous needle biopsies of the vastus lateralis as previously described (18). In each subject, one biopsy was performed under basal conditions before initiating the clamp study and a second was performed in the contralateral leg after $3 \mathrm{~h}$ of steady-state hyperinsulinemia. In some subjects, the second biopsy was performed 30 min after initiating the insulin infusion. To perform the biopsy, a side-cutting needle (Poppler and Sons, New Hyde Park, NY) was inserted through a small incision in locally anesthetized skin $(2 \%$ lidocaine), $\sim 10-15 \mathrm{~cm}$ above the patella on the anterolateral thigh, and $\sim 400 \mathrm{mg}$ muscle tissue was retrieved. This tissue was then blotted on sterile cloth, placed immediately into liquid $\mathrm{N}_{2}$, and stored at $-80^{\circ} \mathrm{C}$. The time between the actual biopsy and snap-freezing of the tissue in liquid $\mathrm{N}_{2}$ was $5-10 \mathrm{~s}$.

\section{Muscle membrane subfractionation}

Frozen vastus lateralis muscle tissue $(\sim 400 \mathrm{mg})$ was placed in $5 \mathrm{vol}$ of buffer ( $\mathrm{pH} 7.6)$ at $4^{\circ} \mathrm{C}$ containing $10 \mathrm{mM}$ Tris- $\mathrm{HCl}, 0.25 \mathrm{M}$ sucrose,

Table I. Clinical Characteristics

\begin{tabular}{|c|c|c|c|c|c|c|}
\hline Subgroup & Number/Gender & Age & Body mass index & Fasting glucose & Fasting insulin & Glucose uptake* \\
\hline & & years & $\mathrm{kg} / \mathrm{m}^{2}$ & $m M$ & $p M$ & $\mathrm{mg} / \mathrm{kg} / \mathrm{min}$ \\
\hline \multicolumn{7}{|l|}{ Protocol 1} \\
\hline Controls & $1 \mathrm{~F} / 4 \mathrm{M}$ & $41 \pm 2$ & $22 \pm 1$ & $5.1 \pm 0.2$ & $29 \pm 12$ & $16.2 \pm 1.1$ \\
\hline Type 2 diabetes & $2 \mathrm{~F} / 2 \mathrm{M}$ & $44 \pm 2$ & $38 \pm 4$ & $13.0 \pm 1.0$ & $169 \pm 53$ & $7.4 \pm 0.5$ \\
\hline \multicolumn{7}{|l|}{ Protocol 2} \\
\hline Insulin-sensitive & $2 \mathrm{~F} / 8 \mathrm{M}$ & $35 \pm 2$ & $26 \pm 2$ & $4.9 \pm 0.1$ & $24 \pm 7$ & $18.1 \pm 2.0$ \\
\hline Insulin-resistant & $2 \mathrm{~F} / 8 \mathrm{M}$ & $34 \pm 3$ & $26 \pm 2$ & $5.0 \pm 0.2$ & $44 \pm 16$ & $12.1 \pm 1.6$ \\
\hline Type 2 diabetes & $3 \mathrm{~F} / 4 \mathrm{M}$ & $43 \pm 1$ & $35 \pm 4$ & $12.1 \pm 1.5$ & $118 \pm 31$ & $9.2 \pm 1.1$ \\
\hline
\end{tabular}

*Glucose uptake represents maximally insulin-stimulated rates of whole body glucose uptake assessed during hyperinsulinemic euglycemic clamps and normalized per kg of lean body mass. In protocol 2, nondiabetic subjects were designated as insulin-sensitive or insulin-resistant based on glucose uptake $>$ of $<15 \mathrm{mg} / \mathrm{kg} / \mathrm{min}$, which was the median value. 
and protease inhibitors including $5 \mu \mathrm{g} / \mathrm{ml}$ leupeptin, $5 \mu \mathrm{g} / \mathrm{ml}$ aprotinin, $1 \mu \mathrm{g} / \mathrm{ml}$ pepstatin, and $400 \mu \mathrm{mol} /$ liter fluoride. The tissue was homogenized first using a Polytron (Brinkmann, Westbury, NY) for five 5 -s bursts at a setting of 5 and then with 10 up-and-down strokes of a motor-driven Teflon pestle in a glass homogenization tube (Thomas Scientific, Swedesboro, NJ). The homogenate was centrifuged at $1,000 \times g$ at $4^{\circ} \mathrm{C}$ for $10 \mathrm{~min}$, and the supernatant was saved. The resulting pellet was resuspended in the buffer, rehomogenized with the glass homogenization tube and Teflon pestle as described above, and recentrifuged at $1,000 \times g$ at $4^{\circ} \mathrm{C}$ for $10 \mathrm{~min}$. The resulting supernatant was combined with the first supernatant and together constituted postnuclear muscle homogenate. The postnuclear homogenate was then processed using either of two protocols for membrane subfractionation.

Protocol 1 (equilibrium sucrose density gradient centrifugation). In each subject, postnuclear homogenates from both basal and maximally insulin-stimulated muscle were centrifuged at $9,000 \times g$ for $10 \mathrm{~min}$, and the resulting supernatants were then centrifuged at $190,000 \times g$ for $60 \mathrm{~min}$. The pellet from each 190,000- $\times$-g centrifugation (crude membranes) was resuspended in buffer and applied to a discontinuous sucrose density gradient containing 25,28 , and $32 \%$ sucrose solutions, respectively. The gradient was centrifuged in a swinging-bucket rotor at $150,000 \times g$ for $16 \mathrm{~h}$ to achieve equilibrium separation of membrane subfractions. Membranes were collected at each sucrose gradient interface, washed by dilution in buffer, and then centrifuged at $190,000 \times g$ for $90 \mathrm{~min}$. The resulting pellets (membrane subfractions) were resuspended in buffer and utilized for immunoblot analyses. All steps were conducted at $4^{\circ} \mathrm{C}$. This method is a modification of that previously used to demonstrate insulin-mediated translocation of glucose transporters in rat muscle (29).

Protocol 2 (nonequilibrium sucrose density gradient centrifugation). The postnuclear homogenates from basal and insulin-stimulated muscle were immediately applied to discontinuous density gradients containing 25,28 , and $32 \%$ sucrose solutions, respectively. Each gradient was centrifuged in a swinging-bucket rotor at $150,000 \times$ $g$ for $3.5 \mathrm{~h}$; this shorter time interval was not sufficient to achieve equilibrium separation of sucrose density gradient subfractions. The sucrose solutions were then separated, diluted in buffer, and centrifuged at $190,000 \times g$ for $90 \mathrm{~min}$. The resulting pellets (membrane subfractions) were resuspended in buffer and utilized for immunoblot analyses. All steps were conducted at $4^{\circ} \mathrm{C}$.

\section{Immunoblot analyses}

For immunoblot analyses, $50-\mu \mathrm{g}$ membrane proteins were solubilized in Laemmli sample buffer (30) and resolved by SDS-PAGE on 1.5$\mathrm{mm}$ slab gels containing $7.5-10 \%$ polyacrylamide. The proteins were then electrophoretically transferred to nitrocellulose filters (31). For immunological detection of GLUT4, nitrocellulose filters were incubated with affinity-purified rabbit antiserum (1:100 dilution) specific for the COOH-terminal segment (44 amino acids) of the GLUT4 transporter cloned from rat skeletal muscle, as previously described (18). Three membrane marker enzymes were also analyzed by immunoblot in the same membrane fractions. Phospholamban is exclusively localized to sarcoplasmic reticulum, and was measured using affinity-purified rabbit antibodies against the canine protein at 1:5,000 dilution (32). Phospholemman was studied as a marker for sarcolemma since it is a chloride channel densely distributed throughout cell surface membranes in cardiac muscle $(33,34)$. Anti-phospholemman antibodies were raised in rabbits against a $\mathrm{COOH}$-terminal peptide (residues 58-72), affinity purified, and used at 1:5,000 dilution (34). The $\alpha-1$ subunit of the dihydropyridine-binding complex (DHP $)^{1}$ was quantified as a $t$-tubule membrane marker using an $\mathrm{mAb}$ (Novus Molecular, Inc., San Diego, CA) at 1:1,000 dilution (35). In

1. Abbreviations used in this paper: DHP, dihydropyridine-binding complex; IRAP, insulin-regulated aminopeptidase. addition, the insulin-regulated aminopeptidase (IRAP) or vp165 (36) was assayed using polyclonal antibodies raised in rabbits against an $\mathrm{NH}_{2}$-terminal peptide $(5 \mu \mathrm{g} / \mathrm{ml}$ dilution $)$, kindly supplied by Dr. S. Keller (Dartmouth College). IRAP is a vesicle protein known to be a constituent of GLUT4-containing vesicles $(36,37)$. Immunoreactivity for GLUT4, phospholamban, and phospholemman was identified using [ ${ }^{125}$ I] protein A, while the immunoreactivity for DHP and IRAP was identified by an enhanced chemiluminescence system (ECL; Amersham Corp., Arlington Heights, IL). Cross-reacting proteins were quantified by autoradiography and scanning densitometry (Molecular Dynamics, Sunnyvale, CA). Parallel experiments were performed to show that each signal in individual subjects was in the linear range of correlation between signal density and amount of protein loaded on gels. A quantitative comparison among the different gels was made possible by including five internal standard lanes on each gel; these lanes contained 50- $\mu$ g membrane samples from two human muscle biopsies and $12.5,50$, and $100 \mu \mathrm{g}$ of crude rat muscle membrane proteins.

\section{Other assays and statistics}

Plasma glucose was measured by the glucose oxidase method with a glucose analyzer (YSI 2300; Yellow Springs Instruments, Yellow Springs, $\mathrm{OH}$ ). Serum insulin levels were measured using a microparticle enzyme immunoassay kit (Abbott Diagnostics, Chicago, IL). Protein was measured by the Coomassie brilliant blue method described by Bradford (38) with crystalline BSA as the standard. Data are means \pm SE. Statistical significance was determined using ANOVA and Student's $t$ test to assess post hoc for group-by-group differences.

\section{Results}

Protocol 1: Equilibrium-density gradient separation of muscle membrane subfractions. To examine whether glucose transport system defects contribute to insulin resistance in type 2 diabetes, we initially studied five lean control subjects and four untreated diabetic patients. As shown in Table I (protocol 1), the mean age in the control and diabetic subgroups was similar; however, the diabetic patients were obese and exhibited fasting hyperglycemia and hyperinsulinemia. Maximally insulin-stimulated glucose uptake rates assessed using the euglycemic clamp technique were reduced by $54 \%$ in the diabetic patients compared with insulin-sensitive control subjects $(P<$ 0.01).

In each subject, vastus lateralis biopsies were obtained under basal conditions and at steady-state serum insulin concentrations that maximally stimulate the glucose transport system. Muscle membranes were subfractionated on sucrose density gradients centrifuged overnight to obtain equilibrium separation of subfractions. Fig. 1 shows membrane distribution of GLUT4 in muscle obtained under basal conditions. In type 2 diabetes, the relative amounts of GLUT4 were decreased by $30 \%$ in the $25 \%$ sucrose density fraction and by $29 \%$ in the $28 \%$ fraction $(P=\mathrm{NS})$. These decrements corresponded with a 2.0 -fold GLUT4 increment in both the $32 \%$ fraction $(P<$ $0.05)$ and in the bottom pellet $(P<0.05)$ compared with controls. Thus, in basal muscle, a greater proportion of GLUT4 was localized in denser membrane fractions in type 2 diabetes. The total tissue content of GLUT4, determined by immunoblot analysis of total membrane fractions (for example, postnuclear homogenate), was similar in the diabetic and nondiabetic control subgroups $(P=\mathrm{NS}$; data not shown) as previously reported $(17,18)$.

The observation that GLUT4 was abnormally localized to denser membrane vesicles in diabetes could be explained by 


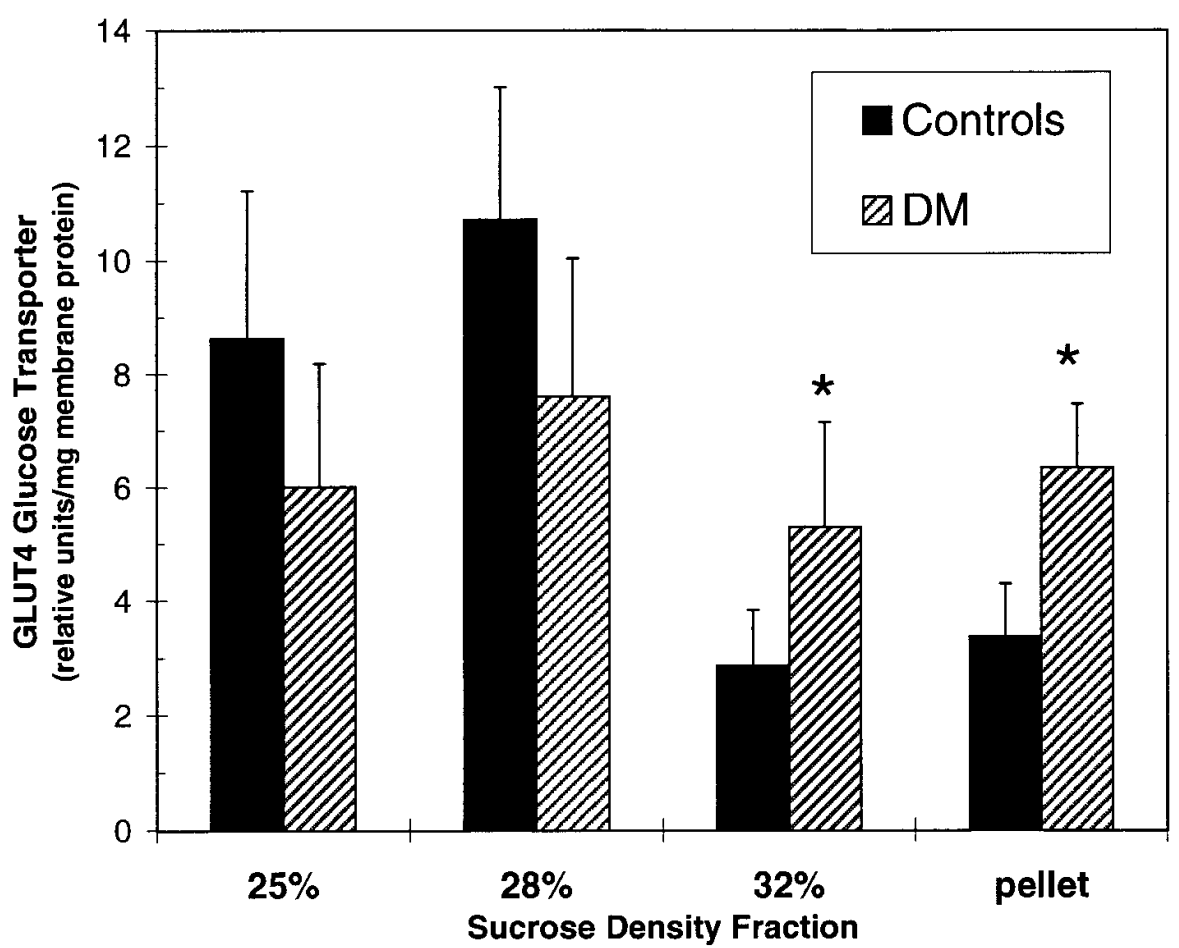

Figure 1. Distribution of GLUT4: equilibrium separation of sucrose density membrane subfractions from basal skeletal muscle. Skeletal muscle biopsies were obtained from insulin-sensitive nondiabetic controls (closed bars) and from type 2 diabetic $(D M)$ patients (open bars) under basal conditions. Muscle was homogenized and subfractionated on discontinuous sucrose gradients until equilibrium separation was achieved. Membrane proteins from each subfraction $(50 \mu \mathrm{g})$ were resolved by SDSPAGE, and reacted with GLUT4 antibodies followed by [ $\left.{ }^{125} \mathrm{I}\right]$ protein A. Relative amounts of GLUT4 were quantified by autoradiography and densitometry, and data represent mean $\pm \mathrm{SE}$ of subgroups delineated in Table I (protocol 1). ${ }^{*} P<0.05$ for $\mathrm{DM}$ versus controls. generalized effects on protein recovery or to nonspecific changes in membrane composition. To address these possibilities, we measured total protein in the postnuclear homogenate and each of the membrane fractions, together with phospholemman, a marker for sarcolemma $(33,34)$, and phospholamban, a marker for sarcoplasmic reticulum (32). Table II delineates data showing recovery of protein and membrane markers. In comparing control and diabetic subgroups, there were no statistically significant differences in the recovery of total protein, phospholemman, or phospholamban in any of the subfractions $(P=\mathrm{NS})$. Thus, major changes in protein recovery or in the subfractionation of sarcolemmal and intracel-

Table II. Protocol 1: Percent Recovery of Protein and Membrane Markers in Muscle Membrane Subfractions

\begin{tabular}{lcccc}
\hline & \multicolumn{4}{c}{ Sucrose gradient fraction } \\
\cline { 2 - 5 } & $25 \%$ & $28 \%$ & $32 \%$ & Pellet \\
\hline Protein recovery & & & & \\
$\quad$ Controls & $8.2 \pm 1.5$ & $3.5 \pm 0.6$ & $2.9 \pm 0.9$ & $20.4 \pm 2.3$ \\
$\quad$ Type 2 diabetes & $9.0 \pm 2.4$ & $3.0 \pm 0.9$ & $3.2 \pm 0.7$ & $19.1 \pm 2.1$ \\
Phospholemman recovery* & & & & \\
$\quad$ Controls & $27.1 \pm 4.0$ & $17.8 \pm 5.9$ & $12.9 \pm 4.1$ & $7.2 \pm 1.6$ \\
$\quad$ Type 2 diabetes & $30.0 \pm 5.6$ & $13.8 \pm 3.0$ & $14.8 \pm 5.8$ & $6.2 \pm 1.8$ \\
Phospholamban recovery* & & & & \\
$\quad$ Controls & $17.4 \pm 2.4$ & $10.5 \pm 2.7$ & $11.4 \pm 4.2$ & $10.6 \pm 4.3$ \\
$\quad$ Type 2 diabetes & $16.1 \pm 2.3$ & $10.7 \pm 3.6$ & $14.1 \pm 4.1$ & $7.3 \pm 1.6$ \\
& & & & \\
\hline
\end{tabular}

*Data represent percent recovery in the membrane fractions of the total present in post-nuclear homogenates. Density gradient subfractions were retrieved after equilibrium separation of membranes (see protocol 1). Muscle biopsies were obtained under basal conditions. lular membranes could not explain differences in the distribution of GLUT4 in basal muscle.

Muscle biopsies were also obtained in the presence of maximal steady-state serum insulin concentrations and processed as described for protocol 1 . In both controls and diabetes, insulin did not affect the amount of GLUT4 in any of the sucrose gradient membrane fractions ( $P=\mathrm{NS}$; data not shown) when compared with basal data (see Fig. 1). Recoveries of protein, phospholemman, and phospholamban were not affected by insulin stimulation $(P=\mathrm{NS}$; data not shown). In these subjects (Table I, protocol 1), the second biopsy was performed after $3 \mathrm{~h}$ of insulin stimulation during the clamp study. In two additional controls, the second biopsy was obtained $30 \mathrm{~min}$ after initiating the insulin infusion, and again, no effect of insulin was observed in these membrane subfractions (data not shown). Thus, insulin-mediated GLUT4 translocation could not be detected in membrane subfractions obtained from sucrose density gradient fractions at equilibrium following prolonged centrifugation.

Protocol 2: Non-equilibrium-density gradient separation of muscle membrane subfractions. In another series of experiments, we prepared sucrose density membrane fractions under non-equilibrium conditions (for example, after a shortened centrifugation period). Muscle biopsies were obtained from a greater number of subjects with normal glucose tolerance to assess glucose transport system defects occurring in the absence of diabetes. These subjects were designated as insulin sensitive or insulin resistant based on maximally insulin-stimulated glucose uptake rates $>$ or $<$ the median value of $15 \mathrm{mg} /$ $\mathrm{kg} / \mathrm{min}$. As shown in Table I, these two nondiabetic subgroups were well matched for mean age and body mass index; however, insulin-sensitive subjects were found to have mean maximal glucose uptake rate $50 \%$ higher than that observed in the insulin-resistant subgroup $(P<0.01)$. Seven type 2 diabetic pa- 


\section{$\mathrm{kDa}$ 69 \\ Figure 2. Immunoblot of GLUT4 glucose transporters in muscle membranes: effects of in- sulin in non-equilibrium sucrose density membrane subfractions. Muscle biopsies were obtained

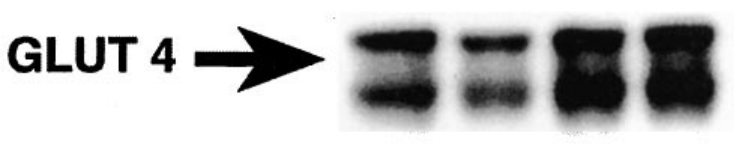 $=$ "4"

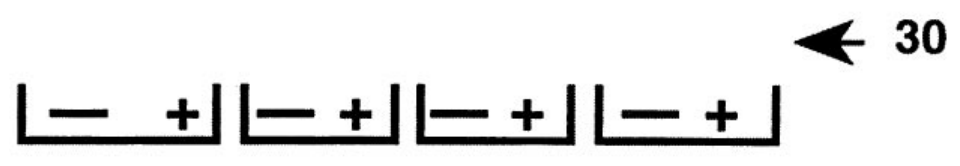 Sucrose
fraction $25 \%$ $28 \%$ $32 \%$ pellet under basal and insulin-stimu- lated conditions from an insulin- sensitive individual. Muscle was homogenized and subfraction- ated on discontinuous sucrose gradients under non-equilib- rium conditions with a shortened centrifugation time. Membrane proteins $(50 \mu \mathrm{g})$ from each subfraction were resolved by SDS-PAGE, and reacted with GLUT4 antibodies followed by $\left[{ }^{125} \mathrm{I}\right]$ protein $\mathrm{A}$. The figure repre- sents an autoradiograph. The lo- cation of molecular weight markers is shown on the right.}

tients were also studied with higher mean age and body mass index; they were uniformly insulin resistant.

As before, muscle biopsies were obtained under basal and hyperinsulinemia conditions, however, the major change from protocol 1 was that sucrose density gradients were centrifuged for only $3.5 \mathrm{~h}$ and subfractions retrieved before equilibrium separation. Relative amounts of GLUT4 were assessed in each membrane subfraction, and an immunoblot from an insulinsensitive subject is shown in Fig. 2. Fig. 3 shows mean GLUT4 amounts detected in the 25 and $32 \%$ sucrose gradient fractions, respectively, in all subjects. Insulin infusion produced small (12-13\%) GLUT4 decrements in the $25 \%$ sucrose fraction that were not statistically significant (all subgroups $P=$ NS). In the $32 \%$ sucrose fraction, insulin increased GLUT4, however, the response was notably different among the subgroups. In the insulin-sensitive subgroup, insulin led to a twofold increase in mean GLUT4 levels in the $32 \%$ sucrose fraction $(P<0.01)$. In both insulin-resistant and type 2 diabetic subjects, the amounts of GLUT4 in the $32 \%$ fraction from in- sulin-stimulated muscle were not significantly different from basal GLUT4 levels ( $P=$ NS). GLUT4 data (not shown) in the $28 \%$ fraction paralleled results observed in the $25 \%$ fraction (mean basal and insulin-stimulated values were 10.5 \pm 0.9 and $10.1 \pm 0.8 \mathrm{U} / \mathrm{mg}$ membrane protein in insulin-sensitive subjects, $10.3 \pm 1.7$ and $8.8 \pm 1.8$ in insulin resistant, and $6.6 \pm 1.2$ and $6.9 \pm 1.1$ in type 2 diabetic subgroups), whereas data in the bottom pellet were analogous to those in the $32 \%$ fraction (mean basal and insulin-stimulated values were $1.5 \pm 0.5$ and $3.1 \pm 0.8$ in insulin sensitive, $2.2 \pm 0.5$ and $2.4 \pm 0.6$ in insulinresistant patients, and $4.0 \pm 0.9$ and $4.0 \pm 1.1$ in diabetes). These differences in GLUT4 localization could not be explained by nonspecific or generalized effects on membrane subfractionation, as demonstrated in Table III. Recoveries of protein, phospholemman, phospholamban, and the $\alpha-1$ subunit of the DHP ( $t$-tubule marker) were similar among insulin sensitive, insulin resistant, and type 2 diabetic subgroups in each sucrose gradient fraction from both basal and insulin-stimulated muscle $(P=\mathrm{NS})$.

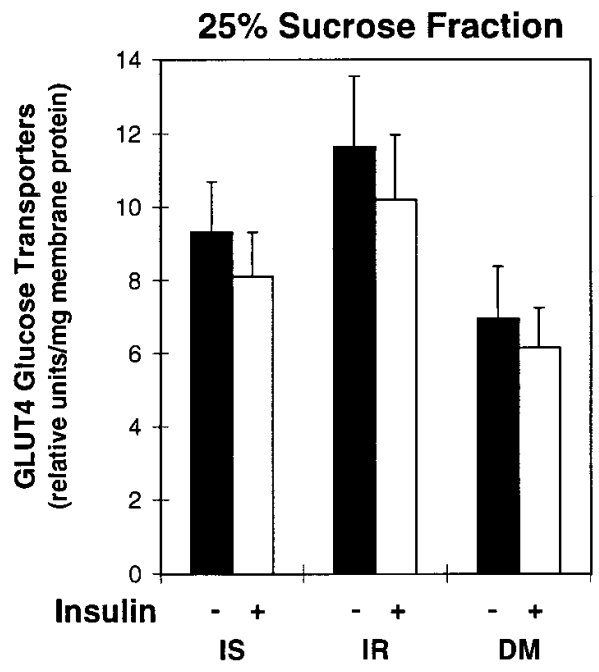

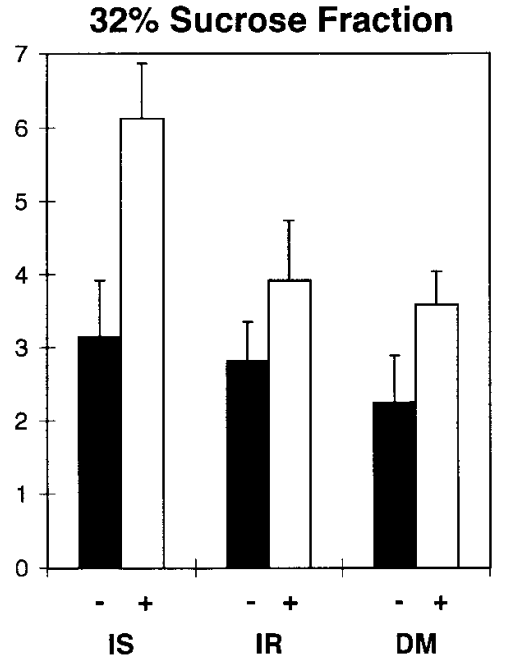

Figure 3. Effects of insulin on GLUT4 in muscle membrane subfractions from insulin-sensitive, insulin-resistant, and type 2 diabetic subgroups. Muscle biopsies were obtained under basal (closed bar) and insulin-stimulated (open bar) conditions from insulin sensitive (IS) and resistant $(I R)$ subjects based on maximal glucose disposal rates $>$ or $<15 \mathrm{mg} / \mathrm{kg}$ lean body mass/min, respectively. Type 2 diabetics $(D M)$ were also studied. Non-equilibrium sucrose density membrane subfractions were obtained, and subjected to immunoblot analyses as described for Fig. 2. GLUT4 was quantified by autoradiography and densitometry, and mean \pm SE levels are shown in the $25 \%$ (left) and $32 \%$ (right) sucrose density subfractions corresponding to subgroups delineated in Table I (protocol 2). 
Table III. Protocol 2: Percent Recovery of Protein and Membrane Markers in Muscle Membrane Subfractions

\begin{tabular}{lcccc}
\hline & \multicolumn{4}{c}{ Sucrose gradient fraction } \\
\cline { 2 - 5 } & $25 \%$ & $28 \%$ & $32 \%$ & Pellet \\
\hline Protein recovery* & & & & \\
$\quad$ Insulin-sensitive & $4.9 \pm 0.8$ & $3.4 \pm 1.2$ & $2.2 \pm 0.5$ & $4.6 \pm 1.0$ \\
Insulin-resistant & $4.4 \pm 0.7$ & $2.7 \pm 0.3$ & $2.6 \pm 0.4$ & $5.7 \pm 1.0$ \\
Type 2 diabetes & $3.9 \pm 0.7$ & $2.2 \pm 0.3$ & $2.6 \pm 1.0$ & $4.8 \pm 1.0$ \\
Phospholemman recovery* & & & & \\
$\quad$ Insulin-sensitive & $28 \pm 6$ & $12 \pm 4$ & $8 \pm 3$ & $7 \pm 2$ \\
Insulin-resistant & $30 \pm 9$ & $12 \pm 3$ & $12 \pm 4$ & $6 \pm 3$ \\
Type 2 diabetes & $27 \pm 4$ & $14 \pm 3$ & $8 \pm 2$ & $9 \pm 3$ \\
Phospholamban recovery* & & & & \\
Insulin-sensitive & $10 \pm 2$ & $10 \pm 1$ & $5 \pm 1$ & $9 \pm 2$ \\
Insulin-resistant & $11 \pm 1$ & $11 \pm 4$ & $6 \pm 2$ & $10 \pm 4$ \\
Type 2 diabetes & $12 \pm 4$ & $12 \pm 3$ & $6 \pm 2$ & $9 \pm 3$ \\
DHP* & & & & \\
Insulin-sensitive & $18 \pm 1$ & $31 \pm 3$ & $16 \pm 2$ & $9 \pm 2$ \\
Insulin-resistant & $21 \pm 1$ & $36 \pm 3$ & $16 \pm 3$ & $8 \pm 2$ \\
Type 2 diabetes & $16 \pm 2$ & $30 \pm 3$ & $16 \pm 2$ & $10 \pm 1$ \\
& & & & \\
\hline
\end{tabular}

*Data represent percent recovery in the membrane fractions of the total present in postnuclear homogenates. Density gradient subfractions were retrieved before equilibrium separation of membranes could occur (see protocol 2). Data from basal and insulin-stimulated muscle were similar and were combined to obtain the mean values.

GLUT4 vesicles also contain IRAP or vp165, which translocates together with GLUT4 to the cell surface $(36,37)$. To further test for abnormalities in GLUT4 vesicle traffic, relative amounts of IRAP were assessed in the muscle membrane subfractions by immunoblot analysis; the data are shown in Table IV. The data generally demonstrate the same two abnormalities in insulin resistant subgroups that were observed for GLUT4. First in basal muscle, 2.6-4.3 times more IRAP is localized in denser membrane vesicles recovered in the bottom pellet of the gradient in both insulin-resistant and diabetic subgroups than in insulin-sensitive subjects. Second, in insulinsensitive subjects, insulin increased IRAP by 1.85 -fold in the $28 \%$ sucrose fraction, whereas this effect was absent in both insulin-resistant nondiabetic and diabetic subgroups. Thus, similar to GLUT4, IRAP is redistributed to a denser membrane compartment and does not translocate in response to insulin in insulin resistant subjects. The discrepancy that insulin augments GLUT4 in the $32 \%$ sucrose fraction and IRAP in the $28 \%$ fraction in the insulin sensitive subgroup may be explained by the fact that not all cellular GLUT4 is located in IRAP-containing vesicles $(39,40)$ and/or that different functional pools of intracellular GLUT4 vesicles contain variable amounts of IRAP relative to GLUT4 (41).

The GLUT4 increment in the $32 \%$ fraction is a quantitative marker for insulin sensitivity. To examine the relationship between GLUT4 changes in the 32\% fraction (protocol 2) and insulin sensitivity, we calculated the insulin-mediated increase over basal in each individual subject. Mean data are shown in Fig. 4. The GLUT4 increment in the insulin sensitive group was 2.82 -fold, which represents a $182 \%$ increase over basal values; this was approximately three times higher $(P<0.01)$ than
Table IV. Effects of Insulin on the IRAP (vp165) in Muscle Membrane Subfractions from Insulin-sensitive, Insulin-resistant, and Type 2 Diabetic Subgroups

\begin{tabular}{lllcc}
\hline & \multicolumn{4}{c}{ Sucrose gradient fraction } \\
\cline { 2 - 5 } & \multicolumn{1}{c}{$25 \%$} & \multicolumn{1}{c}{$28 \%$} & $32 \%$ & Pellet \\
\hline Insulin-sensitive & & & & \\
$\quad$ Basal & $6.5 \pm 6$ & $3.3 \pm 0.5$ & $3.4 \pm 1.1$ & $3.8 \pm 1.2$ \\
$\quad$ Insulin & $5.2 \pm 1.1$ & $6.1 \pm 1.3^{*}$ & $3.9 \pm 1.3$ & $3.8 \pm 0.7$ \\
$\quad$ Insulin-resistant & & & & \\
$\quad$ Basal & $3.3 \pm 0.9$ & $4.4 \pm 0.8$ & $4.0 \pm 1.0$ & $9.7 \pm 1.2^{\ddagger}$ \\
$\quad$ Insulin & $3.6 \pm 0.7$ & $4.6 \pm 1.1$ & $4.8 \pm 1.0$ & $10.2 \pm 6^{\ddagger}$ \\
$\quad$ Type 2 diabetics & & & & \\
$\quad$ Basal & $3.2 \pm 0.8$ & $4.5 \pm 0.4$ & $5.2 \pm 0.6$ & $16.3 \pm 1.3^{\ddagger}$ \\
$\quad$ Insulin & $3.9 \pm 0.8$ & $4.4 \pm 0.7$ & $4.7 \pm 0.4$ & $17.8 \pm 3.2^{\ddagger}$ \\
& & & & \\
\hline
\end{tabular}

Skeletal muscle biopsies were subjected to non-equilibrium sucrose density membrane subfractionation (protocol 2), and subfractions were subjected to immunoblot analysis using antibodies against IRAP. The data represent the mean $\pm \mathrm{SE}$ of relative levels in the various subgroups. $* P<0.05$ when compared with value in basal muscle from insulin-sensitive subjects. ${ }^{\ddagger} P<0.05$ when compared with values in the pellet from insulin-sensitive subjects.

the $54 \%$ increase in insulin-resistant subjects (1.54-fold) and the $63 \%$ increase in Type 2 Diabetes (1.63-fold). Thus, the ability of insulin to augment GLUT4 in the $32 \%$ fraction was markedly impaired even in normoglycemic insulin-resistant individuals, and could not be attributed to antagonistic effects of hyperglycemia on insulin action $(4,42)$ (for example, "glucose toxicity"). To assess the relationship between the insulin effect in the $32 \%$ fraction and glycemia-independent insulin resistance, the GLUT4 increment was correlated with the maximally stimulated glucose uptake rate in nondiabetic subjects (for example, insulin-sensitive plus -resistant subjects). Fig. 5 shows that there was a positive and statistically significant correlation between these two variables. Two aspects of the data indicate that the association between insulin resistance and low GLUT4 increment was independent of obesity. First, BMI was not correlated with GLUT4 increment $(P=\mathrm{NS})$, and, second, the GLUT4 increment was found to be greater in insulin sensitive than in insulin-resistant subjects (Fig. 4) despite the fact that these subgroups were well matched for body mass index (Table I).

\section{Discussion}

In this study, sucrose density gradients were used to separate membrane subfractions under two conditions, at equilibrium and under non-equilibrium conditions after a shortened centrifugation time. Data using membrane fractions obtained under non-equilibrium conditions demonstrated that insulin augments GLUT4 by 2.8 -fold in one sucrose gradient fraction (32\% sucrose), concomitant with a trend towards reduction in other fractions ( $25 \%$ and $28 \%$ sucrose) in insulin-sensitive individuals. These observations support the conclusion that insulin stimulates glucose transport via translocation of GLUT4 to sarcolemma in human muscle.

Investigators have relied on either subcellular membrane 


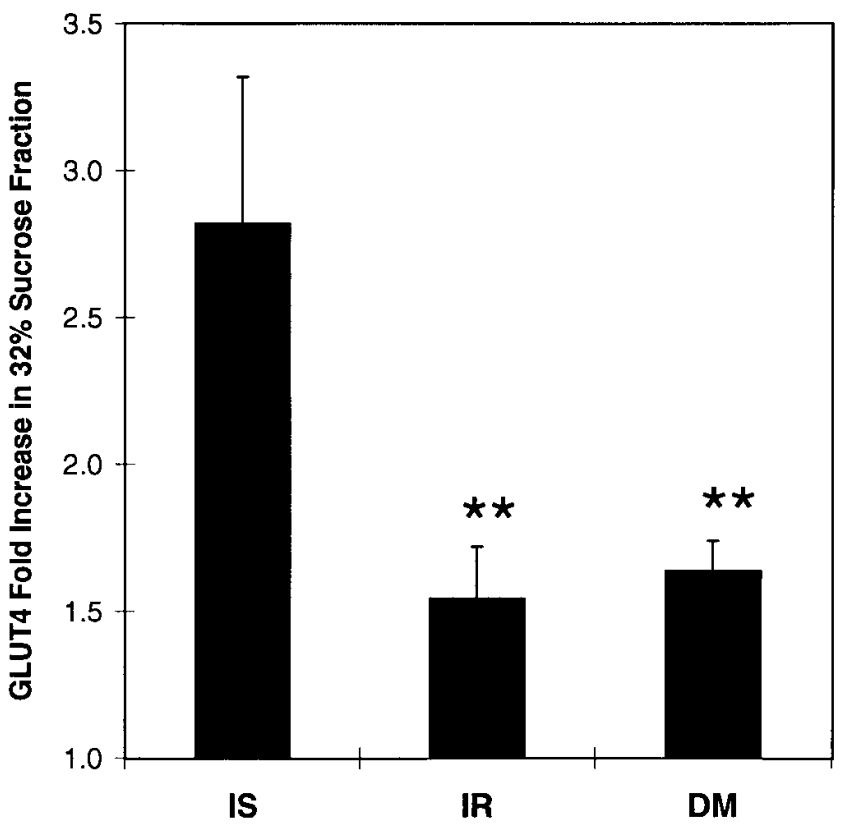

Figure 4. Insulin-mediated increase in GLUT4 in the $32 \%$ sucrose density membrane subfraction. The GLUT4 increment in the $32 \%$ sucrose density membrane subfraction from insulin-stimulated muscle was calculated as fold increase over basal in each individual (see Fig. 3). Data represent the mean $\pm \mathrm{SE}$ of increments in muscle from insulin-sensitive $(I S)$, insulin-resistant $(I R)$, and type 2 diabetic $(D M)$ individuals. ${ }^{* *} P<0.01$ compared with IS.

fractionation or immunocytochemistry of basal and insulinstimulated muscle to resolve GLUT4 translocation. Subcellular fractionation of skeletal muscle has proven much more difficult than it has in adipocytes; the effectiveness of this biochemical approach in muscle has been limited by poor recovery of membrane markers, a high degree of contamination among membrane subfractions, and lack of a marker that defines the intracellular pool. In rat muscle, several groups of investigators have persevered and obtained generally concordant data showing that insulin leads to two- to threefold enrichment of transporters in a sarcolemma-enriched fraction and a decrease in these parameters in microsomes $(29,43,44)$. Biochemical study of human muscle is more limited. In our experiments using membranes subfractionated to equilibrium, insulin stimulation did not affect GLUT4 distribution in control or diabetic subjects, and translocation was not apparent, suggesting that GLUT4 donor and acceptor membranes are both present in the same fractions. Similarly, Lund et al. (45) failed to demonstrate insulin-mediated GLUT4 translocation in both non-diabetic controls and type 2 diabetic patients. Guma et al. (46) were later able to show highly variable responses with a mean $60 \%$ GLUT4 increase in one sucrose density membrane subfraction together with $50 \%$ decrease in another in muscle from normoglycemic individuals. The immunocytochemical approach has demonstrated recruitment of intracellular GLUT4 to sarcolemma in both rats and humans (4), although there has been controversy regarding the intracellular localization in basal muscle (for example, tubulovesicular structures, subsarcolemmal vesicles, transverse tubules and terminal cisternae-triads). In humans, Friedman et al. (47) used immunogold EM to show that insulin moved GLUT4 from tubulovesicular structures and triads to sarcolemma, and Kelley et al. (6), using confocal laser scanning microscopy found that insulin doubled GLUT4 label at sarcolemma. Thus, despite some points of controversy and inconsistency, previous data point to GLUT4 translocation as an important mechanism of transport stimulation in human muscle.

The current data also provide biochemical evidence that

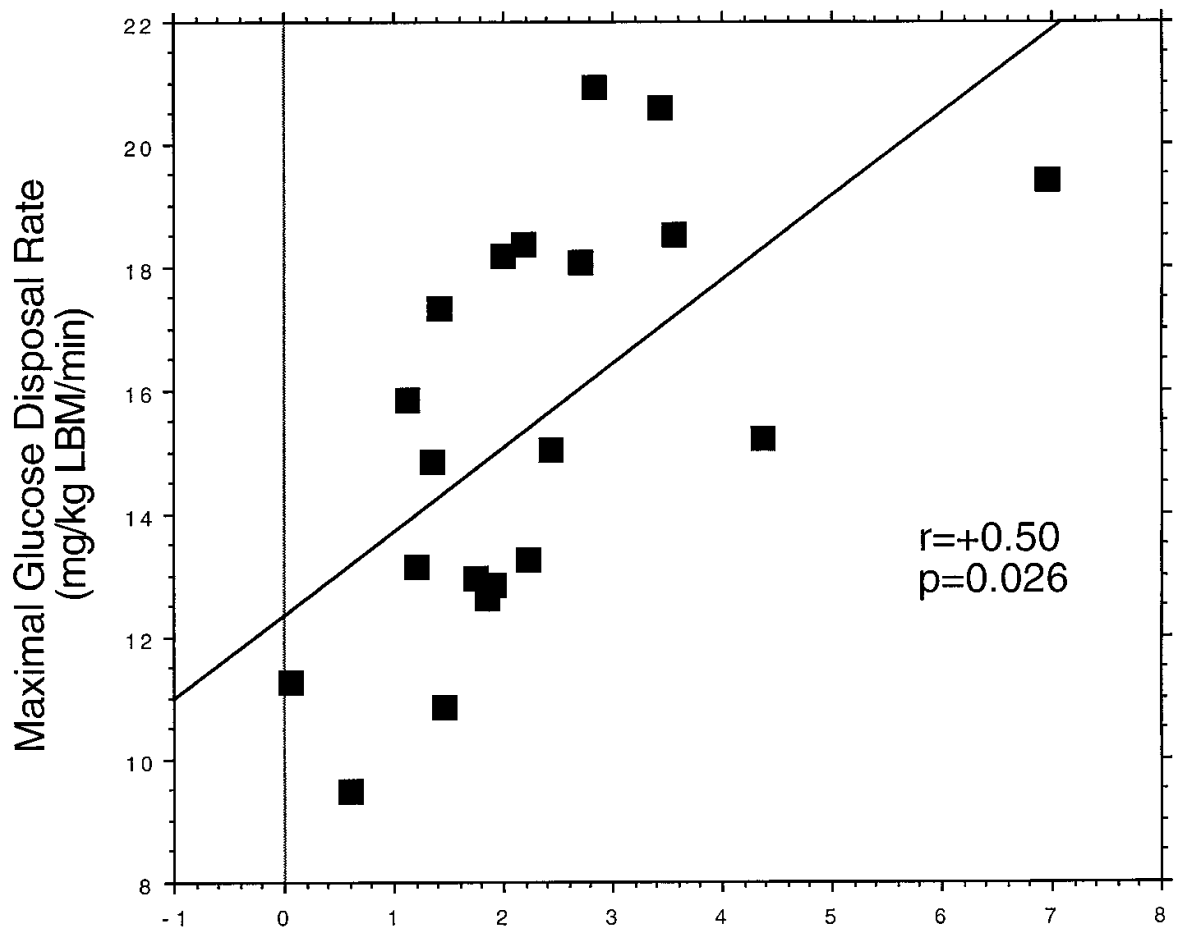

GLUT 4 Fold-Increase in 32\% Sucrose Fraction
Figure 5. The relationship between the GLUT4 increase in the $32 \%$ sucrose density membrane fraction and insulin sensitivity. In muscle from individual normoglycemic subjects (IS and $I R$ from Fig. 4), the fold increase in GLUT4 (insulin-stimulated over basal) in the $32 \%$ sucrose density membrane subfraction was correlated with the maximally stimulated in vivo glucose disposal rate normalized per kilogram lean body mass. 
impaired GLUT4 translocation in muscle can explain human insulin resistance. Such a translocation defect has been inferred as a cause of human insulin resistance, without much supportive empirical data, ever since muscle GLUT4 expression was found to be normal in these patients $(17,18)$. Insulin's ability to increase GLUT4 in the $32 \%$ sucrose fraction was significantly correlated with maximally-stimulated glucose disposal rates, an in vivo measure of insulin sensitivity that largely reflects insulin action in muscle. Thus, the GLUT4 increase in the $32 \%$ sucrose fraction either represents GLUT4 recruitment to sarcolemma or is a quantitative marker for this process. When the data were considered in the context of subject subgroups, the insulin-mediated GLUT4 increment was markedly reduced in both insulin-resistant and type 2 diabetic individuals $(\sim 1.5$-fold above basal) when compared with the 2.8 fold increase in insulin-sensitive subjects. These differences were not accompanied by differences in organelle marker proteins in the various membrane subfractions, indicating that the abnormalities were specific for GLUT4. The data demonstrate a defect in insulin-mediated GLUT4 translocation that is equally severe in insulin-resistant patients with and without diabetes. Recent reports indicate that more intracellular GLUT4 may translocate to $t$-tubule membranes than surface sarcolemma (48-50). The 32\% sucrose fraction contains ample quantities of the protein marker for $t$-tubules as well as phospholemman which is densely distributed along sarcolemma in cardiac muscle $(33,34)$, but so do other fractions. The data imply that GLUT4 is recruited to a particular domain of sarcolemma that is enriched in the $32 \%$ sucrose fraction under these conditions, and this step is impaired in insulin-resistant subjects.

The current biochemical results are consistent with recent immunocytochemical data reported by Kelley et al. (6), who showed reduced GLUT4 staining associated with sarcolemma following insulin stimulation in obese and type 2 diabetic patients. However, our data indicate that the translocation defect is not a function of obesity since insulin sensitive and resistant subgroups were well-matched for body mass index and percent body fat. Zierath et al. (19) observed a 1.6-fold GLUT4 increase in a plasma membrane fraction from nondiabetic controls but no effect of insulin in type 2 diabetics. The current data, using non-equilibrium membrane subfractions, show a much larger increment (2.8-fold) in insulin-sensitive nondiabetics. Analysis of the current data, together with those of Kelley et al. (6) and Zierath et al. (19), illustrates an important point when studying insulin resistance in nondiabetic and diabetic subjects. There are large individual differences in insulin sensitivity among normoglycemic "controls," and this variation is largely independent of measures of generalized obesity (for example, both lean and obese subjects can be either very insulin sensitive or resistant) (51-53). Therefore, nondiabetic subjects should not be considered as a homogeneous group in comparison with type 2 diabetic patients, and obesity cannot effectively be used to identify which nondiabetic subjects are insulin resistant. One explanation for the larger insulin effect (2.8-fold GLUT4 increment in the insulin-sensitive subgroup) is that we have defined a control group on the basis of insulin sensitivity distinct from insulin-resistant nondiabetics. The translocation defect in the current paper is pertinent to the larger obesity-independent component of insulin resistance. Furthermore, the study of separate insulin-sensitive, -resistant, and diabetic subgroups can distinguish defects induced by hy- perglycemia. The translocation defect associated with insulin resistance cannot be attributed to effects of hyperglycemia or "glucose toxicity" $(4,42)$, since it is equally severe in the normoglycemic insulin resistant and type 2 diabetic subgroups. Thus, impaired translocation could contribute to insulin resistance in prediabetic individuals and contribute to the insulin resistance syndrome (for example, syndrome X) (1). The relevance of the translocation defect to the pathogenesis of the insulin resistance syndrome is a hypothesis deserving further study.

An additional important observation was that the translocation defect was accompanied by a second abnormality in GLUT4 subcellular localization demonstrable even in basal muscle. This novel abnormality was clearly observed after equilibrium separation of sucrose density membrane subfractions but was also evident in the experiments using non-equilibrium subfractions. Greater amounts of GLUT4 were targeted to dense membrane vesicles (recovered in the $32 \%$ sucrose fraction and bottom pellet), and lesser amounts recovered in lighter vesicles (recovered in 25 and $28 \%$ sucrose fractions), in insulin-resistant subgroups compared with insulinsensitive controls. This accumulation of GLUT4 in denser membrane vesicles was specific for GLUT4 and did not affect sarcolemma and sarcoplasmic reticulum membrane markers. A similar pattern of abnormalities in insulin-resistant subjects was observed for IRAP (or vp165), a constituent of GLUT4containing vesicles $(36,37)$. In basal muscle from both insulin resistant nondiabetic and diabetic individuals, IRAP was redistributed to dense membrane vesicles recovered in the sucrose gradient pellet relative to that observed in the insulin-sensitive subgroup. In addition, insulin was able to augment IRAP in the $28 \%$ sucrose fraction in the insulin-sensitive subjects but had no effect in the insulin resistant subgroups. An important difference in insulin-sensitive subjects was that insulin increased GLUT4 in the $32 \%$ fraction and IRAP in the $28 \%$ fraction. The data do not explain the reason for this difference. However, it is known that different functional pools of intracellular GLUT4 vesicles in rat skeletal muscle and other cells contain variable proportions of IRAP relative to GLUT4 (3941). These observations provide possible explanations of why translocation of IRAP and GLUT4 were resolved in different sucrose density fractions. In general, the data are complementary. The important points are that GLUT4 and IRAP accumulated in or were targeted to a dense membrane compartment, and this abnormality was associated with a defect in insulin-mediated translocation in insulin-resistant subjects.

This combination of defects in GLUT4 traffic and targeting and translocation is analogous to a pattern we have previously observed in human adipocytes. In insulin-resistant omental adipocytes from women with gestational diabetes mellitus, a much greater proportion of cellular GLUT4 was localized in dense membrane vesicles (cofractionating with plasma membranes and high-density microsomes) in basal cells compared with that in nondiabetic gravidas, and there was little or no GLUT4 recruitment from microsomes to plasma membrane in response to insulin (16). In subcutaneous adipocytes from type 2 diabetic patients, it is difficult to accurately assess subcellular trafficking due to the profound reduction in GLUT4 expression (15). More recently, we (54) have been able to localize GLUT4 vesicles in membrane fractions via study of IRAP (36, 37). In adipocytes from normal controls, IRAP and GLUT4 were observed to translocate from low-density microsomes to 
plasma membrane in response to insulin. In contrast, in diabetes, IRAP accumulated in vesicles cofractionating with plasma membrane and high-density microsomes, and this was associated with impaired insulin-mediated translocation of IRAP. Based on current and previous data, we propose that a defect in GLUT4 trafficking and targeting causes human insulin resistance and is common to both skeletal muscle and adipocytes. This defect leads to GLUT4 accumulation in a dense membrane compartment from which transporters are unable to be recruited to cell surface membranes. This mechanism of insulin resistance is operative in adipocytes but is relegated to secondary importance by a profound reduction in GLUT4 expression in type 2 diabetes. However, this mechanism predominates in skeletal muscle where normal expression of GLUT4 is maintained.

In the current experiments, the non-equilibrium sucrose density gradient fractions show a high degree of cross contamination for sarcolemma (phospholemman), sarcoplasmic reticulum (phospholamban), and $t$-tubule ( $\alpha-1$ subunit of the dihydropyridine binding complex) markers. Even though there is extensive overlap of marker proteins, we have defined experimental conditions in human muscle showing that $(i)$ under basal conditions, GLUT4 accumulates or is redistributed to a dense membrane compartment in insulin resistant individuals compared with insulin sensitive subjects; and (ii) in response to insulin, specific changes in GLUT4 localization are correlated with glucose uptake activity and are impaired in insulin resistant subjects. The data constitute strong operational evidence for combined defects in GLUT trafficking and translocation in skeletal muscle as a cause for human insulin resistance.

The biochemical basis of defects in GLUT4 trafficking remains to be defined. A translocation defect per se could be due to either impaired insulin signal transduction or lie intrinsic to the glucose transporter effector system. Reduced insulin receptor tyrosine kinase activity, phosphorylation of insulin receptor substrate-1, and activation of PI-3-kinase have been observed in skeletal muscle from obese and type 2 diabetic patients (20). While signal transduction abnormalities may be contributory, it should be emphasized that the translocation defect is associated with accumulation of GLUT4 in dense membrane vesicles in basal tissue. Thus, the defect in cellular targeting or trafficking of GLUT4 is independent of insulin. The clear implication is that the abnormality in basal GLUT4 targeting and trafficking is a distinct defect intrinsic to the glucose transport system, which is then mechanistically linked to impaired translocation. This is in contrast to other known cellular $(55)$ and animal models $(42,56)$ of insulin resistance that involve isolated translocation defects without evidence that GLUT4 trafficking is disturbed under basal conditions. GLUT4 vesicle trafficking is constitutive, highly regulated, multicompartmental $(4,21)$, and involves multiple proteins that also direct exocytosis and endocytosis of synaptic neurosecretory vesicles (22-25). It is, therefore, feasible that defects at any of several steps could alter trafficking and targeting of GLUT4 to produce the novel pattern of abnormalities observed in human skeletal muscle. It will be important to elucidate molecular mechanisms leading to GLUT4 accumulation in denser membrane vesicles, the identity of this membrane compartment, and how this alteration impairs translocation in response to insulin. These defects would have important implications for both treatment and prevention of type 2 diabetes and the insulin resistance syndrome.

\section{Acknowledgments}

We gratefully acknowledge Dr. Larry Jones of Indiana University for the gift of antibodies to phospholemman and phospholamban and Dr. Susanna Keller of Dartmouth College for antibodies to IRAP. We thank Dr. Alan J. Gross, Ph.D., of the Department of Biometry and Epidemiology of Medical University of South Carolina for assistance with statistics, John Bercik of the Medical University of South Carolina as General Clinical Research Center Systems Manager for database management, and Ms. Pamela Beasley for typing the manuscript.

This work was supported by National Institutes of Health grants DK-38764 and DK-42469, the Merit Review program and Research Service of the Department of Veterans Affairs, General Clinical Research Centers grants M01-RR-750 and M01-RR-1070, and the Human Biology Core of the Diabetes Research and Training Center at Indiana University (P60-DK-20542).

\section{References}

1. Reaven, G.M. 1995. Pathophysiology of insulin resistance in human disease. Physiol. Rev. 75:473-486.

2. Warram, J.H., B.C. Martin, A.S. Krolewski, J.S. Soeldner, and C.R. Kahn. 1990. Slow glucose removal rate and hyperinsulinemia precede the development of type II diabetes in the offspring of diabetic parents. Ann. Intern. Med. 113:909-915.

3. DeFronzo, R.A., E. Jacot, E. Jequier, E. Maeder, and J.P. Felber. 1981. The effect of insulin on the disposal of intravenous glucose: results from indirect calorimetry and hepatic and femoral venous catheterization. Diabetes. 30: 1000-1007.

4. Garvey, W.T., and M. Birnbaum. 1993. Cellular insulin action and insulin resistance. In Bailliere's clinical endocrinology and metabolism: insulin resistance and disease. E. Ferrannini, editor. Bailliere Tindall, London. 785-873.

5. Bonadonna, R.C., S. Del Prato, M.P. Sacomani, E. Bonora, G. Gulli, E. Ferrannini, D. Bier, C. Cobelli, and R.A. DeFronzo. 1993. Transmembrane glucose transport in skeletal muscle of patients with non-insulin-dependent diabetes. J. Clin. Invest. 92:486-494.

6. Kelley, D.E., M.A. Minton, S.C. Watkins, J.-A. Simoneau, F. Jadali, A. Fredrickson, J. Beattie, and R. Theriault. 1996. The effect of non-insulin-dependent diabetes mellitus and obesity on glucose transport and phosphorylation in skeletal muscle. J. Clin. Invest. 97:2705-2713.

7. Katz, A., B.L. Nyomba, and C. Bogardus. 1988. No accumulation of glucose in human skeletal muscle during euglycemic hyperinsulinemia. Am. J. Physiol. 255:E942-E945.

8. Ziel, F.H., N. Venkatesan, and M.B. Davidson. 1988. Glucose transport is rate limiting for skeletal muscle glucose metabolism in normal and STZinduced diabetic rats. Diabetes. 37:885-890.

9. Shulman, G., D. Rothman, T. Jue, and P. Stein. 1990. Quantitation of muscle glycogen synthesis in normal subjects with NIDDM by ${ }^{13} \mathrm{C}$ nuclear magnetic resonance spectroscopy. N. Engl. J. Med. 322:223-228.

10. Butler, P.C., E.J. Kryshak, M. Marsh, and R.A. Rizza. 1990. Effect of insulin on oxidation of intracellularly and extracellularly derived glucose in patients with NIDDM: evidence for primary defect in glucose transport and/or phosphorylation but not oxidation. Diabetes. 39:1371-1380.

11. Kelley, D.E., J.P. Reilly, T. Veneman, and L.J. Mandarino. 1990. Effects of insulin on skeletal muscle glucose storage, oxidation, and glycolysis in humans. Am. J. Physiol. 258:E923-E929.

12. Henry, R.R., B. Gumbiner, T. Flynn, and A.W. Thorburn. 1990. Metabolic effects of hyperglycemia and hyperinsulinemia on fate of intracellular glucose in NIDDM. Diabetes. 39:149-156.

13. Dohm, G.L., E.B. Tapscott, W.J. Pories, D.J. Dabbs, E.G. Flickinger, D. Meelheim, T. Fushiki, S.M. Atkinson, C.W. Elton, and J.F. Caro. 1988. An in vitro human muscle preparation suitable for metabolic studies: decreased insulin stimulation of glucose transport in muscle from morbidly obese and diabetic subjects. J. Clin. Invest. 82:486-494.

14. Andreasson, K., D. Galuska, A. Thorne, T. Sonnenfeld, and H. Wallberg-Henriksson. 1991. Decreased insulin-stimulated 3- $O$-methylglucose transport in vitro incubated muscle strips from type II diabetic subjects. Acta. Physiol. Scand. 142:255-260.

15. Garvey, W.T., L. Maianu, T.P. Huecksteadt, M.J. Birnbaum, M.J. Molina, and T.P. Ciaraldi. 1991. Pretranslational suppression of a glucose transporter protein causes cellular insulin resistance in non-insulin-dependent diabetes mellitus and obesity. J. Clin. Invest. 87:1072-1081.

16. Garvey, W.T., L. Maianu, J.-H. Zhu, J.A. Hancock, and A.M. Golichowski. 1993. Multiple defects in the adipocyte glucose transport system cause cellular insulin resistance in gestational diabetes: heterogeneity in the number and a novel abnormality in subcellular localization of GLUT4 glucose transporters. Diabetes. 42:1773-1785. 
17. Pederson, O., J.F. Bak, P.H. Andersen, S. Lund, D.E. Moller, J.S. Flier, and B.B. Kahn. 1990. Evidence against altered expression of GLUT1 or GLUT4 in skeletal muscle of patients with obesity or NIDDM. Diabetes. 39: $865-870$

18. Garvey, W.T., L. Maianu, J.A. Hancock, A.M. Golichowski, and A. Baron. 1992. Gene expression of GLUT4 in skeletal muscle from insulin-resistant patients with obesity, IGT, GDM, and NIDDM. Diabetes. 41:465-475.

19. Zierath, J.R., L. He, A. Guma, E.O. Wahlstrom, A. Klip, and H. Walberg-Henriksson. 1996. Insulin action on glucose transport and plasma membrane GLUT4 content in skeletal muscle from patients with NIDDM. Diabetologia. 39:1180-1189.

20. Goodyear, L.J., F. Giorgino, L.A. Sherman, J. Carey, R.J. Smith, and G.L. Dohm. 1995. Insulin receptor phosphorylation, insulin receptor substrate-1 phosphorylation, and phosphatidylinositol 3-kinase activity are decreased in intact skeletal muscle strips from obese subjects. J. Clin. Invest. 95:2195-2204.

21. Czeck, M.P. 1995. Molecular actions of insulin on glucose transport. Annu. Rev. Nutr. 15:441-471.

22. Cheatham, B., A. Volchuk, C.R. Kahn, L. Wang, C.J. Rhodes, and A. Klip. 1996. Insulin-stimulated translocation of GLUT4 glucose transporters requires SNARE-complex proteins. Proc. Natl. Acad. Sci. USA. 93:15169-15173.

23. Timmers, K.I., A.E. Clark, M. Omatsu-Kanbe, S.W. Whiteheart, M.K. Bennett, G.D. Holman, and S.W. Cushman. 1996. Identification of SNAP receptors in rat adipose cell membrane fractions and in SNARE complexes coimmunoprecipitated with epitope-tagged $\mathrm{N}$-ethylmaleimide-sensitive fusion protein. Biochem. J. 320:429-436.

24. Sollner, T.H., and J.E. Rothman. 1996. Molecular machinery mediating vesicle budding, docking, and fusion. Cell Struct. Funct. 21:407-412.

25. Sudhof, T.C. 1995. The synaptic vesicle cycle: a cascade of protein-protein interactions. Nature. 375:645-653.

26. National Diabetes Data Group. 1979. Classification and diagnosis of diabetes mellitus and other categories of glucose intolerance. Diabetes. 28:10391057.

27. Garvey, W.T., J.M. Olefsky, J. Griffin, R.F. Hamman, and O.G. Kolterman. 1985. The effect of insulin treatment on insulin secretion and insulin action in type II diabetes mellitus. Diabetes. 34:222-234.

28. Baron, A., M. Laakso, G. Brechtel, and S.V. Edelman. 1991. Reduced capacity and affinity of skeletal muscle for insulin-mediated glucose uptake in non-insulin-dependent diabetic subjects. J. Clin. Invest. 87:1186-1194.

29. Klip, A., T. Ramlal, D.A. Young, and J.O. Holloszy. 1987. Insulininduced translocation of glucose transporter in rat hindlimb muscles. FEBS (Fed. Eur. Biochem. Soc.) Lett. 224:224-230.

30. Laemmli, U.K. 1970. Cleavage of structural proteins during the assembly of the head of bacteriophage T4. Nature. 227:680-685.

31. Towbin, H., T. Staehelin, and J. Gordon. 1979. Electrophoretic transfer of proteins from polyacrylamide gels to nitrocellulose sheets: procedure and some applications. Proc. Natl. Acad. Sci. USA. 76:4350-4354.

32. Jorgensen, A.O., and L.R. Jones. 1986. Localization of phospholamban in slow but not fast canine skeletal muscle fibers: an immunocytochemical and biochemical study. J. Biol. Chem. 261:3775-3781.

33. Palmer, C.J., B.T. Scott, and L.R. Jones. 1991. Purification and complete sequence determination of the major plasma membrane substrate for c-AMPdependent protein kinase and protein kinase $\mathrm{C}$ in myocardium. J. Biol. Chem. 266:11126-11130.

34. Moorman, J.R., C.J. Palmer, J.E. John, M.E. Durieux, and L.R. Jones. 1992. Phospholemman expression induces a hyperpolarization-activated chloride current in Xenopus oocytes. J. Biol. Chem. 267:14551-14554.

35. Morton, M.E., J.M. Caffrey, A.M. Brown, and S.C. Froehner. 1988. Monoclonal antibody to the alpha 1-subunit of the dihydropyridine-binding complex inhibits calcium currents in BC3H1 myocytes. J. Biol. Chem. 263:613616.

36. Keller, S.R., H.M. Scott, C.C. Mastick, R. Aebersold, and G.E. Lienhard. 1995. Cloning and characterization of a novel insulin-regulated membrane aminopeptidase from GLUT4 vesicles. J. Biol. Chem. 270:23612-23618.

37. Kandror, K.V., and P.F. Pilch. 1994. gp160, a tissue specific marker for insulin-activated glucose transport. Proc. Natl. Acad. Sci. USA. 91:8017-8021.

38. Bradford, M. 1976. A rapid and sensitive method for the quantitation of microgram quantities of protein utilizing the principle protein-dye bonding. Anal. Biochem. 72:248-254.

39. Martin, S., J.E. Rice, G.W. Gould, S.R. Keller, J.W. Slot, and D.E. James. 1997. The glucose transporter GLUT4 and the aminopeptidase vp165 colocalise in tubulo-vesicular elements in adipocytes and cardiomyocytes. $J$. Cell. Sci. 110:2281-2291.

40. Filippis, A., S. Clark, and J. Proietto. 1998. Possible role for gp160 in constitutive but not insulin-stimulated GLUT4 trafficking: dissociation of gp160 and GLUT4 localization. Biochem. J. 330:405-411.

41. Aledo, J.C., L. Lavoie, A. Volchuk, S.R. Keller, A. Klip, and H.S. Hundal. 1997. Identification and characterization of two distinct intracellular GLUT4 pools in rat skeletal muscle: evidence for an endosomal and an insulinsensitive GLUT4 compartment. Biochem. J. 325 (Pt. 3):727-732.

42. Baron, A.D., J.-S. Zhu, J.-H. Zhu, H. Weldon, L. Maianu, and W.T. Garvey. 1995. Glucosamine induces insulin resistance in vivo by affecting GLUT4 translocation in skeletal muscle: implications for glucose toxicity. $J$. Clin. Invest. 96:2792-2801.

43. Sternlicht, E., R.J. Barnard, and G.K. Grimditch. 1988. Mechanism of insulin action on glucose transport in rat skeletal muscle. Am. J. Physiol. 254: E633-E638.

44. Hirshman, M.F., L.J. Goodyear, L.J. Wardzala, E.D. Horton, and E.S Horton. 1990. Identification of an intracellular pool of glucose transporters from basal and insulin-stimulated rat skeletal muscle. J. Biol. Chem 265:987991.

45. Lund, S., H. Vestergaard, P.H. Andersen, O. Schmitz, L.B. Gotzsche and O. Pedersen. 1993. GLUT4 content in plasma membrane of muscle from patients with non-insulin-dependent diabetes mellitus. Am. J. Physiol. 265: E889-E897.

46. Guma, A., J.R. Zierath, H. Wallberg-Henriksson, and A. Klip. 1995. Insulin induces translocation of GLUT4 glucose transporters in human skeletal muscle. Am. J. Physiol. 268:E613-E622.

47. Friedman, J.E., R.W. Dudek, D.S. Whitehead, D.L. Downes, W.R Frisell, J.F. Caro, and G.L. Dohm. 1991. Immunolocalization of glucose transporter GLUT4 within human skeletal muscle. Diabetes. 40:150-154.

48. Munoz, P., M. Rosemblatt, X. Testar, M. Palacin, G. Thoidis, P.F. Pilch, and A. Zorzano. 1995. The T-tubule is a cell-surface target for insulin-regulated recycling of membrane proteins in skeletal muscle. Biochem. J. 312 (Pt. 2):393400

49. Dombrowski, L., D. Roy, B. Marcotte, and A. Marette. 1996. A new procedure for the isolation of plasma membranes, T-tubules, and internal membranes from skeletal muscle. Am. J. Physiol. 270:E667-E676.

50. Wang, W., P.A. Hansen, B.A. Marshall, J.O. Holloszy, and M. Mueckler. 1996. Insulin unmasks a $\mathrm{COOH}$-terminal GLUT4 epitope and increases glucose transport across $t$-tubules in skeletal mucle. J. Cell Biol. 135:415-430.

51. Hollenbeck, C., and G.M. Reaven. 1987. Variations in insulin-stimulated glucose uptake in healthy individuals with normal glucose tolerance. $J$. Clin. Endocrinol. Metab. 64:1169-1173.

52. Bogardus, C., and S. Lillioja. 1992. Pima Indians as a model to study the genetics of NIDDM. J. Cell. Biochem. 48:337-343.

53. Garvey, W.T., L. Maianu, G. Brechtel, A. Johnson, K. Sunblad, and D. Hardin. 1994. Muscle GLUT4 expression is a familial trait and determines insulin sensitivity in humans independent of obesity. Diabetes. 43 (Suppl. 1):69a

54. Maianu, L., S. Keller, and W.T. Garvey. 1996. Fat and muscle express a common abnormality in translocation/trafficking of GLUT4/vp165 containing vesicles in NIDDM. Diabetes. 45 (Suppl. 2):157a

55. Garvey, W.T., J.M. Olefsky, S. Matthaei, and S. Marshall. 1987. Glucose and insulin coregulate the glucose transport system in primary cultured adipocytes: a new mechanism of insulin resistance. J. Biol. Chem. 262:189-197.

56. Kin, P.A., E.D. Horton, M.F. Hirshman, and E.S. Horton. 1992. Insulin resistance in obese Zucker rat (fa/fa) skeletal muscle is associated with a failure of glucose transporter translocation. J. Clin. Invest. 90:1568-1575. 